\title{
MathMap: Supporting Exploratory Problem Solving with Algebra
}

\author{
Matthew T Beaudouin-Lafon \\ University of California, San Diego \\ mbeaudouinlafon@ucsd.edu
}

\author{
Haijun Xia \\ University of California, San Diego \\ haijunxia@ucsd.edu
}

\begin{abstract}
Tools that support problem-solving in mathematics tend to focus on reaching a solution directly. In practice, it is common to go down paths that do not obviously lead to the solution. This part of the process should be reflected in the tools students use to help them better learn problem-solving strategies. MathMap is an application designed to help high-school students learn how to solve algebraic problems by encouraging them to use multiple strategies, maintain the history of previous attempts, and allows them to meaningfully compare methods with other students.
\end{abstract}

\section{CCS CONCEPTS}

- User Interface Design; • Collaborative and Social Computing;

\section{KEYWORDS}

Math, Exploration, Computer Algebra Systems

\section{ACM Reference Format:}

Matthew T Beaudouin-Lafon and Haijun Xia. 2021. MathMap: Supporting Exploratory Problem Solving with Algebra. In The Adjunct Publication of the 34th Annual ACM Symposium on User Interface Software and Technology (UIST '21 Adjunct), October 10-14, 2021, Virtual Event, USA. ACM, New York, NY, USA, 4 pages. https://doi.org/10.1145/3474349.3480226

\section{INTRODUCTION}

In mathematics, algebra is used to represent and solve problems. When a problem is written in algebraic notation, it can be manipulated according to rigorous rules until it represents a solution. A sizable portion of the K-12 curriculum is dedicated to learning and applying this notation, which indicates it is both important and challenging to learn. Students first learn the basic mechanics of the notation (e.g., fractions, order of operations, solving equations, etc.), and later practice using algebra better understand specific domains (e.g., polynomials, trigonometry, calculus, etc.). We direct our attention to the latter case.

The medium in which notation is embedded affects how it is used. Today, pen and paper are the standard medium for high-school mathematics. It can flexibly support text, diagrams and simple plots. However, solving algebraic problems using pen and paper is cognitively demanding. For example, to "cancel out $\mathrm{x}$ in this

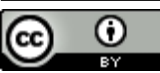

This work is licensed under a Creative Commons Attribution International 4.0 License.

UIST '21 Adjunct, October 10-14, 2021, Virtual Event, USA

(c) 2021 Copyright held by the owner/author(s).

ACM ISBN 978-1-4503-8655-5/21/10

https://doi.org/10.1145/3474349.3480226 fraction" students must mentally apply the transformation and write the new expression character by character. This detour is error prone and can distract from learning high-level problem-solving strategies. In the past few decades, Computer Algebra Systems (CAS) have grown in popularity to address this problem. A student simply needs to input the problem, and the CAS can produce an answer. This can be useful to solve sub-problems, view alternate representations, and check answers [6][7], but while the CAS can show the steps it took, the student has neither practiced taking those steps, nor attempted other strategies.

We believe that to support learning the problem-solving strategies, the medium should minimize the effort in mechanically applying algebraic rules such that the student can focus on solving higher level problems. Such a medium should also encourage the students to reflect on their decisions and explore alternative strategies.

We are developing MathMap, a tool that helps students learn how to solve algebraic problems. With MathMap, users can select the rules they wish to apply to an algebraic expression, and it will automatically execute the transformation. The problem-solving process is represented by a tree where nodes are expressions, and edges are transformations from one node to the next. Within MathMap, users can interact with the tree structure to explore the problem space and develop higher level understanding of strategies for solving algebraic problems. Specifically, users can branch off from any node to spatially explore alternative strategies, temporally explore previous attempts to reflect on their problem-solving process and communally explore how other students approach the same problem.

\section{RELATED WORK}

MathMap builds upon prior work on problem solving pedagogy to facilitate learning of algebra. De Jong and Fergusson-Hessler [4] define four types of knowledge, including procedural knowledge as "actions or manipulations that are valid within a domain," and strategic knowledge that "helps students organize their problemsolving process by directing which stages they should go through to reach a solution". Students develop these kinds of knowledge to solve high-level problems by comparing different strategies via trial and error, reflecting on previous operations, and learning from their peers through collaboration [5]. MathMap supports these different ways of learning by enabling students to spatially, temporally, and communally explore the problem space.

Previous work to support algebra has primarily focused on using interaction techniques to simplify the use of notation. MathDeck [2] simplifies equation input in LaTeX with mixed representations and by promoting the reuse of sub-expression. MathBrush [1] uses handwriting recognition to interpret user input and provides an interface 


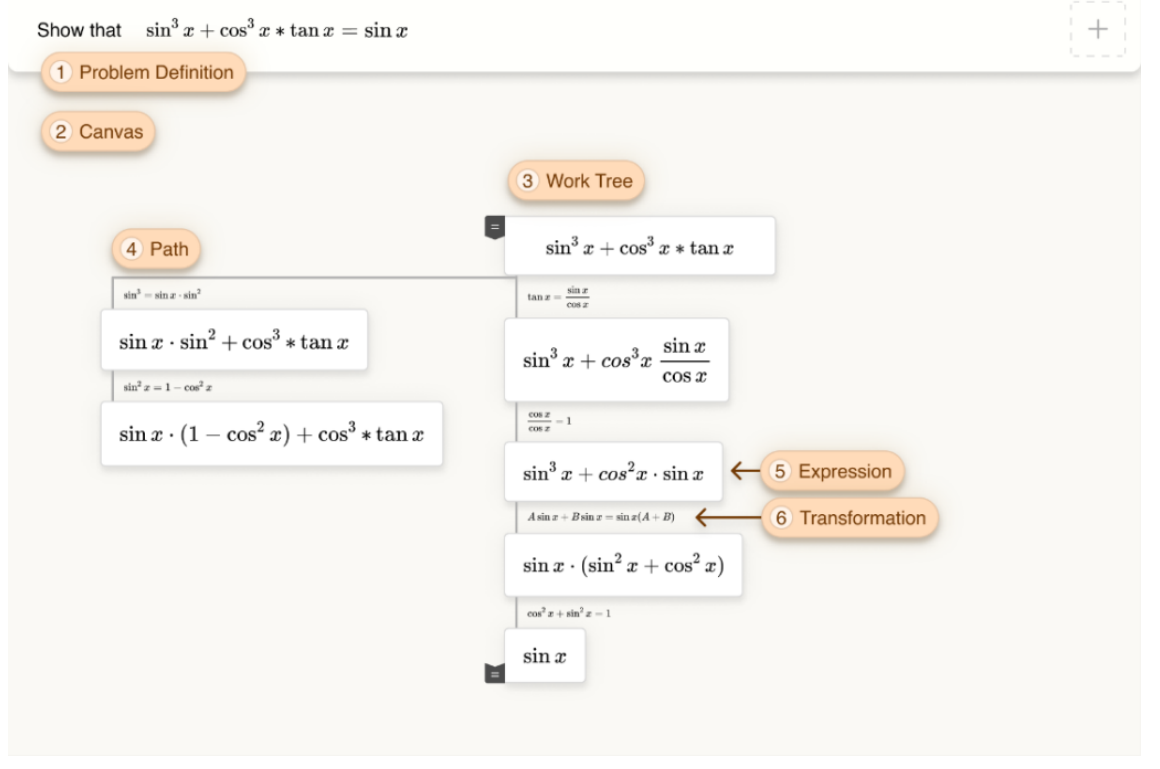

Figure 1: Overview of MathMap's interface.

for applying standard CAS operations. Hands-on Math [3] also recognizes handwritten math; and proposes pen and touch interaction techniques to perform algebraic transformations, such as factoring and simplifying fractions. While Hands-on Math successfully reduces friction in using algebraic notation, it does not explicitly support higher-level decision making or exploration. MathMap also uses CAS to apply individual transformation but goes further to encourage exploration and learning high-level problem solving.

\section{DESIGN OF MATHMAP}

MathMap is an application that helps students learn how to solve algebraic problems. We focus our attention on proving trigonometric identities, taking derivatives and integrals of functions. These problems often require applying multiple different algebraic techniques and are hence well suited for exploratory work.

The interface is composed of two main sections: a fixed problem definition and a canvas (Fig. 1). The canvas hosts Work Trees, collections of expressions connected by transformations. The user drags an expression from the problem definition and builds the tree by sequentially applying transformations to these expressions. The Work Tree branches when the user applies a new transformation to an expression earlier in the tree, effectively exploring a different path. The problem is considered solved when there is a path from the problem to the solution.

Organizing expressions and transformations into the work tree enables a few interactions to support exploration. Spatial Exploration refers to applying different strategies to a problem and understand which is the best solution. As the student works through problems, they will develop a mental model that maps problems to strategies. Through Temporal Exploration, the student reflects on the strategies they have already used. Finally, Communal Exploration refers to comparing the strategies explored by other students and understanding how they approached the problem.

\subsection{Spatial Exploration}

To encourage the user to attempt different strategies, adding and branching the work tree should be simple. A work tree is initiated either by dragging an expression from the problem statement or dragging a sub-expression out of an expression card. The work tree is designed for the users to easily try different strategies and see where they lead. To learn effectively, the student must retain ownership of each decision. Hence, to apply a transformation, the user selects the target subexpression, and MathMap shows possible transformations (Fig. 2.1 and 2.2) by pattern matching the selection to transformations stored in its bank. The user selects the appropriate transformation, and it is applied to a new expression card connected to the previous one (Fig 2.3). The user saw other possibilities, which may have inspired other approaches. Our goal is to strike a balance between maintaining the user's ownership of the problem-solving decisions while softly suggesting other routes; without guaranteeing its success. This way, the user is still forced to apply strategies and identify which work, but without false negatives due to arithmetic mistakes. As a result, building the work tree is an intentional exploratory process.

\subsection{Temporal Exploration}

In addition to exploring paths forward, students should also reflect on paths taken. MathMap shows how the expressions change by displaying the transformation between each card. However, following these transformations is burdensome. When the user selects a sub-expression, MathMap traces it across cards and transformations to help the user understand how it evolved in this path (Fig 3.1). Additionally, when the user selects a transformation in the tree, the system displays the other suggestions that were originally shown (Fig 3.2). This helps the user remember the context in which they 

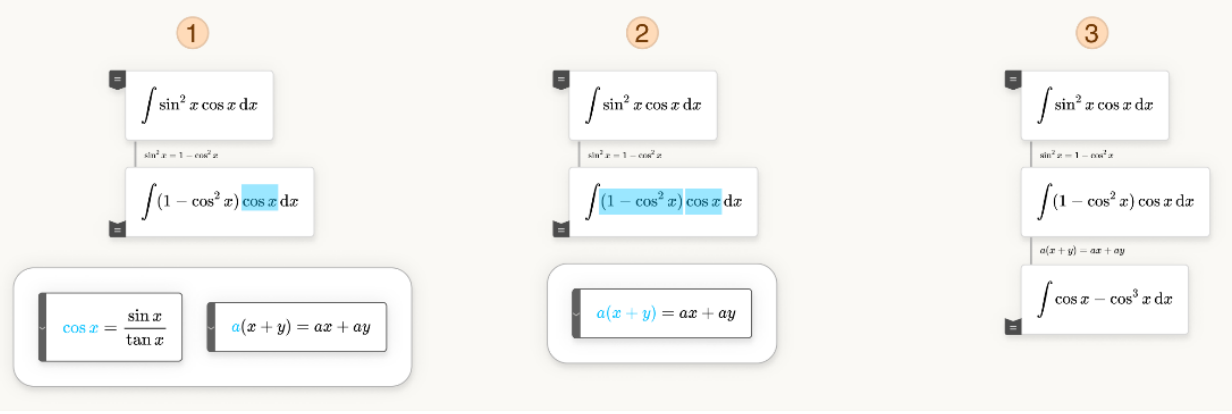

Figure 2: Building the work tree. The user begins by (1) selecting the subexpression they intend to transform (blue highlight), (2) selects additional subexpression to better filter suggested transformations and (3) clicks on the transformation, which the system applies and adds the resulting expression to the work tree.

1

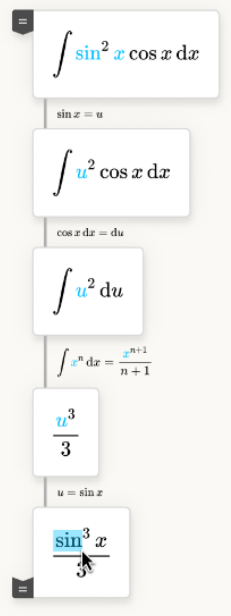

2

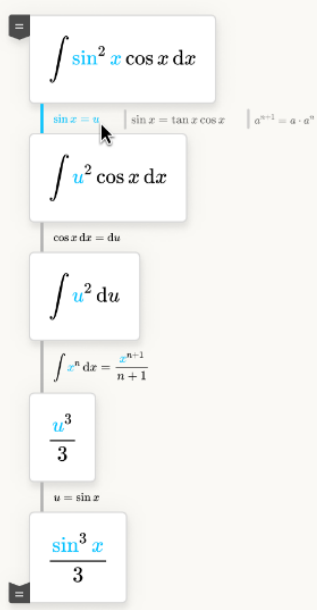

Figure 3: Temporal Exploration. (1) When a user selects a sub-expression, it is highlighted in the rest of the work tree and tracked through expressions. (2) The user can also select a transformation and see the other transformations that were suggested at this step.

chose a transformation. These interactions help the user explore the impact of their previous decisions.

\subsection{Communal Exploration}

Finally, to better understand the space of possible problem-solving strategies, students may learn from exploring how their classmates solved the same problem. Teachers also benefit from exploring what the class did to identify patterns and adjust future lectures.

When students submit their work, a teacher can view their canvases individually. The shape of the Work Tree provides insight into how the student solved the problem (Fig 4). For example, a straight line from the problem statement to the solution suggests that the student knew and utilized an effective strategy quickly, but a wide tree suggests that they attempted many different strategies without seeing them through. The teacher also gains insight from the Spatial and Temporal Exploration techniques described above.
The teacher can compile the responses from all students into an Aggregate View. Similar paths are overlaid, and parting branches show the percentage of students that used each path (Fig 4.1). Paths are also ordered from most common on the left to least common on the right. Since different students might arrive to the same expression through different paths, the resulting artifact is no longer a tree. Exploring this view helps the teacher understand the breadth of strategies employed and discover common pitfalls. Students can also benefit from learning how others applied transformations using the Aggregate View. They can inspect how transformations are used by other students. Selecting a transformation in the list highlights it in the aggregate work tree (Fig 4.2).

\section{FUTURE WORK}

MathMap is a prototype we developed to probe the design of user interface and interaction techniques to support learning algebra. 


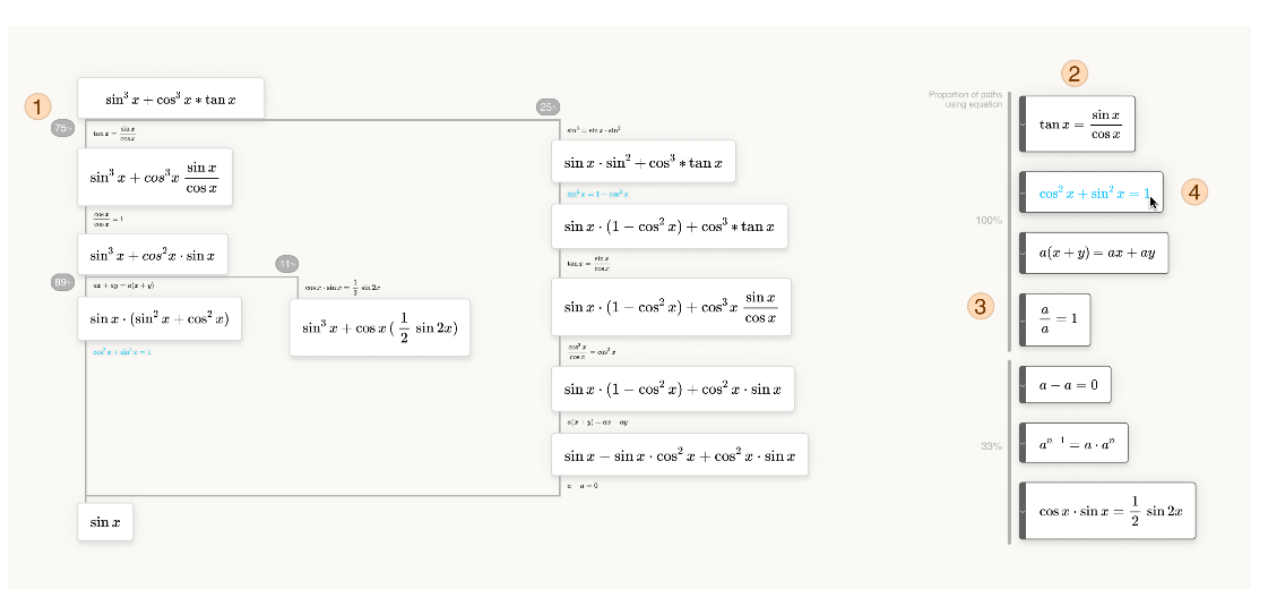

Figure 4: Communal Exploration. The Aggregate View (1) combines work trees from the class and displays what portion of the group used which path. Transformations are also compiled (2) and sorted by usage across paths (3). (4) highlighting transformations in this view shows them in the Aggregate View on the right.

Our future research will benefit from a formative study with educators and students to better understand their pain points and inform the next iteration. We will also study whether MathMap promotes exploration better than pen and paper, the extent to which students brute force their way to a solution, and whether students effectively learn problem-solving strategies. MathMap's scope could also be extended to help students use algebra to solve physics problems and balance chemical equations. However, the ideas behind spatial, temporal, and communal exploration could also be applied to other domains with discrete transformations such as Computer Aided Design (CAD), or creative procedural tasks like designing 3D textures in a node-based editor and building audio effect chains.

\section{CONCLUSION}

We presented MathMap, an application that encourages exploration to solve algebraic problems. The user decides how to solve the problem at each step but is unburdened from managing notational details. Exploration is promoted through interaction with expressions and the layout of the work (spatially), the examination of past attempts (temporally), and comparison with the work of other students (communally).

\section{REFERENCES}

[1] Labahn, G, Lank, E, MacLean, S, Marzouk, M, and Tausky, D. Math-Brush: A System for Doing Math on Pen-Based Devices. IAPR Inter-national Workshop on Document Analysis Systems (2008), 599-606

[2] Yancarlos Diaz, Gavin Nishizawa, Behrooz Mansouri, Kenny Davila, and Richard Zanibbi. 2021. The MathDeck Formula Editor: Interactive Formula Entry Combining LaTeX, Structure Editing, and Search. Extended Abstracts of the 2021 CHI Conference on Human Factors in Computing Systems. Association for Computing Machinery, New York, NY, USA, Article 192, 1-5. DOI:https://doi.org/10.1145/3411763.3451564

[3] Robert Zeleznik, Andrew Bragdon, Ferdi Adeputra, and Hsu-Sheng Ko. 2010. Hands-on math: a page-based multi-touch and pen desktop for technical work and problem solving. Proceedings of the 23nd annual ACM symposium on User interface software and technology. Association for Computing Machinery, New York, NY, USA, 17-26. DOI:https://doi.org/10.1145/1866029.1866035

[4] De Jong, T., \& Ferguson-Hessler, M. G. M. (1996). Types and qualities of knowledge. Educational Psychologist, 31, 105-113.

[5] Bernhard Ertl. 2009. Conceptual and procedural knowledge construction in computer supported collaborative learning. In Proceedings of the 9th international conference on Computer supported collaborative learning - Volume 1 (CSCL'09). International Society of the Learning Sciences, 137-141.

[6] Pierce, Robyn, Herbert, Sandra and Giri, Jason 2004, CAS : student engagement requires unambiguous advantages, in Mathematics education for the third millennium : towards 2010, Mathematics Education Research Group of Australasia, Pymble, N.S.W., pp. 462-469.

[7] Drijvers, P. Students encountering obstacles using a CAS. International fournal of Computers for Mathematical Learning 5, 189-209 (2000). https://doi.org/10.1023/A: 1009825629417 